\title{
The rhythms of infrastructure
}

\section{Mikko Jalas, Jenny Rinkinen \& Antti Silvast}

Mikko Jalas is a senior lecturer at the Aalto University School of Arts, Design and Architecture. His email is mikko.jalas@aalto.fi.

Jenny Rinkinen is a senior research associate at the DEMAND Centre (Dynamics of Energy, Mobility and Demand) at Lancaster University. Her email is jenny.rinkinen@lancaster.ac.uk.

Antti Silvast is a research fellow at the Science, Technology and Innovation Studies unit at the School of Social and Political Science, University of Edinburgh. His email is antit.silvast@ed.ac.uk.

Household technology frequently appears as a set of objects and practices that aim to save time. From cooking to communications, novelties enter the home accompanied by claims of increased efficiency and convenience. The effects of new technology are, however, multidimensional and unanticipated: apart from saving time, they impact on the division of labour, create new expectations and require supportive actions such as maintenance and service (Schwartz Cowan 1983). In this article, we suggest that rhythmicity is a key effect of technology.

We employ recent advances in theories of social practice (Shove et al. 2012), according to which, both technology and time are resources that are integrated in particular ways through enacting practices in everyday life. Our approach is also informed by the claim of Lefebvre ([1974] 1991: 206) that social practice is made up of rhythms of different scales. When combined, these perspectives suggest that energy infrastructures can be understood and analyzed through the rhythmicity that they produce and are embedded in. We thus argue that energy infrastructures organize time and produce collective rhythms.

We begin by defining the concept of an infrastructure and developing approaches to the temporality of infrastructure provisioning. Using these approaches, we move on to discuss the use of electricity grid services and of more isolated domestic wood-based heating systems. In both cases, the normal operation of the system yields particular routinized temporalities which stand in contrast to those that are enacted in the event of failure and underservice by the infrastructure.

The use of electricity, the consequences of eventual blackouts and the measures taken to ensure high quality grid services depend on installed energy technology and its sociotechnical context. We mainly draw on recent data from Finland on which we have previously reported (Rinkinen 2013, 2015; Silvast 2013) but which we now revisit with an eye for the rhythmic effects of energy infrastructures. The main research methodology we used to gather information on security, risk and interruptions in the electricity infrastructure in Finland was multi-sited fieldwork. Our empirical materials include interviews with experts (30 persons) and Finnish lay people who have experienced extended blackouts (15 persons), a consumer survey (115 respondents), and participant observation carried out in two electricity control rooms and electricity security seminars in Finland. Additionally, we draw on around 50 infrastructure and security policy documents, mainly from Finland and Scandinavia, to gain more information about infrastructure risk management practices, especially in the electricity industries.

Concerning the routinization of domestic heating work, the data come from a large set of pre-collected diaries kept at the ethnological archive of the Finnish Literature Society. Of these, we have analyzed approximately 1,000 single diaries, each ranging from 1-10 pages. In addition, we have also drawn on Finnish time use survey data.

\section{From large-scale to distributed infrastructures}

The notion of large-scale technical systems, and particularly infrastructure, informs this article. Hughes, in his classic study of the history of electrification, defines a system as consisting of interconnected parts and components that are connected by a network or structure (Hughes 1983: 5). This simple definition implies that systems are composed of parts that can be managed and steered - frequently centrally. However, this definition excludes those elements that exist in the environment but cannot be steered. Thus the electricity grid is composed of physical components such as transformers and transmission lines, organizations such as utility companies, scientific components such as university education and research programmes, laws and regulation as well as natural resources such as mines (Hughes 1989: 51).

Research on large-scale technical systems began in the 1980s and placed a new emphasis on the key social role of systems such as energy provision (van der Vleuten 2004: 399). One of the central observations emerging from this research was that dispersed technical systems are layered. Technologies shape many other parts of society and adjust to them: it is, for example, feasible to think that organizations, institutions, laws and scientific programmes emerge and are shaped together with those technologies that they aim to govern. Over the past 10 years, several scholars have sought to readdress the premises of this research on large-scale technical systems, but prefer to use the notion of infrastructure. While systems tend to be diffuse, changing little over time, infrastructures are scaled down and locally adapted. As Edwards et al. (2009: 370) claim, 'the actual infrastructures of people's real work lives always involve particular configurations of numerous tools used in locally particular ways'.

\section{Practices as manifestations of infrastructures}

The theory of social practice is an increasingly prominent way to account for human action that is structured by sociotechnical systems and infrastructures (e.g. van Vliet et al. 2005). Social practices such as dwelling, working, and getting from one place to another are activities that human beings enact in pre-given ways. Accordingly, technical systems enable and prompt particular ways and courses of action and inhibit others.

End users are more or less strictly connected to infrastructures via social practices that constitute the taken-for-granted ways of going about everyday life (Southerton et al. 2004; Shove et al. 2015). On the one hand, we have highly regulated and technically structured practices and infrastructures, such as air travel, for example. On the other hand, as a less coercive structure, a practice may offer merely a way of evaluating situations and reasoning proper conduct. Thinking about our empirical case here, the range of options available - from district heating to off-grid solutions for domestic heating - offer alternative ways to relate to public infrastructures and time. 
The work of Lefebvre ([1974] 1991) can be used to add that the structuring or configuration of human activities achieved by infrastructures is: (a) rhythmic and (b) both temporal and spatial. For example, systems of public transportation represent fixed coordinates in time and space, amass people and underlie routinization in everyday life. Hence, social life can be seen to consist of rhythmic reappearances of performances that define and pin down meanings of both temporal (the temporal architecture of social life) and spatial (physical space and architecture) locations.

Lefebvre ([1959] 2002) also suggests that the performance of everyday life always includes an element of blind, child-like playfulness that contrasts with coercion and reasoned, effective and convenient ways of acting. In all cases, practices have a temporal, performative dimension as social structures are realized in human action. Hence, if infrastructures can be thought of as practices and practices are necessarily also performative, we suggest that infrastructures have temporal manifestations in terms of the sets of activities that depend on them and make them needed and at times even critical. Indeed, Edwards (2003) has also stated that infrastructures, although inconspicuous, affect the experience of time in fundamental ways:

infrastructures possess the power to shape human time, shaping the preconditions under which we experience time's structure and its passage ... Societies build infrastructure, of course, but because of their endurance in time, infrastructures then become the more important force in structuring society. (Edwards 2003: 195)

The way that infrastructures affect everyday rhythms has received relatively scant interest (see Silvast et al. 2013). However, the above quote highlights the stabilization and patterning of time as an outcome of infrastructures. This patterning of time is an effect of the co-evolution and mutual adjustment of infrastructures and the practices of everyday life. The infrastructure of private mobility is, for example, tied to patterns of work and systems of food provisioning. As technological novelties are gradually integrated into everyday life and novices turn into routinized users of technology, infrastructure begins to act as a set of self-evident coordinates of daily life. Stable and taken-for-granted infrastructures hence demand and reproduce routines and structure the use of time. These routines and their rhythms are interestingly exposed when infrastructures malfunction.

\section{Regularity and temporal coordination}

In their normal state, infrastructures provide services uninterrupted and unnoticed. Indeed, our data indicate that the reliability of infrastructures is a key political concern. A set of European Union (EU) directives has laid the foundation for the service delivery and security of a national electricity infrastructure (Darby 2012). The Finnish government, for example, passed a new law on electricity markets in 2013, which stipulates that market actors must make plans and prepare for non-normal situations. Moreover, they have to provide instructions to their customers in the event of an interruption to the electricity supply. Suppliers of critical infrastructure services are thus required both to take precautionary measures in order to build resilient systems that enable an uninterrupted supply and to build capacity for quickly restoring services in the eventual case of failure. End users are variously engaged in emergency preparedness. In terms of indoor heating provision for instance, housing permits may, as in Finland for example, require that detached houses have to have auxiliary heating systems. In most cases, end users are, however, only advised to take more modest measures of preparedness such as having bottled water in the house and battery-powered communication devices.

If and when the infrastructure service is reliable enough, it can assert effects on other system parts and become fundamental to the normal procedures of everyday life. Fast communication networks for example, contribute to more mobile patterns of work if, and only if, the service can be relied upon to be available. Yet all infrastructures, and particularly infrastructures that are plagued with failures and interruptions, co-exist with alternative systems of provision and a related alternative patterning of time (Rinkinen 2013; Trentmann 2009). Hence, in a modern city, there are routinized patterns of use of the public transportation service, but at the same time more dormant practices of making do without it exist. In a similar way, even if Finnish households are increasingly electrified and dependent on uninterrupted delivery, ways of managing without electricity also exist.

Blackouts lack a clear temporal structure - or more precisely, they are structured by their irregularity. Although they are frequently connected to weather events, the incidence and duration of blackouts are rarely known in advance. For most of our interviewees, blackouts catch end users by surprise. Such frequent but unanticipated failures and interruptions in service supply create both preparedness and frustration among the interviewees and survey respondents. In addition to the need to prepare, infrastructure failures also create room for more positive emotions: failures such as electricity blackouts interrupt the monotony of continuous service delivery and break down routines. Trentmann (2009: 74) reports a similar finding in relation to a 1965 blackout in the US when people referred to their experiences during the blackout as 'making a holiday'. Hence, in addition to preparing for failures, the new skills and creative coping strategies developed in response, represent a willingness to tolerate such failures (Silvast 2013).

\section{Regulation of the availability of services}

Not all infrastructures are designed to supply services uninterruptedly and at a constant rate. Public transportation services operate on the basis of collective mobility schedules, and the office hours of private businesses and public administration are limited. The availability of some services are regulated to avoid public harm and negative health effects, as in the serving of alcohol in some cases. Even the service of those infrastructures that have been designed for constant operation may be temporally regulated on occasion: during periods of water scarcity, for instance, the supply of water may be limited in order to achieve a critical level of services for all connected households (Trentmann 2009).

Regulated infrastructure services contribute to a particular temporal patterning. The known and anticipated but interrupted availability of services requires that other activities be scheduled accordingly. Such infrastructures can only become opaque and unnoticed background features through a routinization that matches demand with the fluctuating supply.

\section{Weather and the rhythmicity of nature}

Much like regulated infrastructures, the cycles of nature and weather events are fundamental sources of social rhythms (Lefebvre [1974] 1991; Adam 1995), which frequently also reflect energy demand. Daily, monthly and yearly cycles alter outdoor conditions and affect which activities are necessary for individuals to survive and for societies to function. Yet most technological systems serve to isolate us from the cycles of nature. Houses provide shelter and increasingly standardized indoor environments, lighting lessens the effects of the daily and yearly variation of sunlight, and communication networks collapse time and space. The availability and disruption of energy services thus affect the way that we can experience and are exposed to natural rhythms. Blackouts can in this way be understood as 'taking a holiday' from everyday routines, bringing a less 
technically mediated and more direct experience of nature. In other words, during blackouts geophysical rhythms enter the households that are protected and isolated from them during states of normalcy.

There is a further point to make about the weather. The weather is not simply an issue that calls for protection and technologies of encasement (Hitchings \& Lee 2008). Rather, as the popular notion of ecosystem services depicts, human societies are crucially dependent on the functioning of natural systems. Weather events thus act as a kind of infrastructure, providing services for humans, pacing and scheduling activities. If weather is thought of as an infrastructure providing a pervasive rhythmic temporality, technologies of encasement and lighting contribute another layer of infrastructure that lessens and flattens out the cycles of ecosystem services. In general, infrastructures are layered one upon another and partly function to counter the rhythms of one another.

To sum up the way that infrastructures organize time, we need to consider the states of normal operation and those of disruption. Infrastructures as ideal types are opaque and unnoticed (Graham 2012). If and when service availability is limited, infrastructures get ingrained with other rhythms of life whereby the availability of a service subtly matches demand, even if both fluctuate. This is the case in the regulated availability of infrastructure services. The disruption and failure of a single infrastructure service, on the other hand, exposes users to new underlying rhythms that may be of a human making or originate in nature. Blackouts, for example, may lead to the enactment of alternative techniques for keeping warm or cool or may expose humans more directly to outdoor temperatures.

\section{Heating work}

Infrastructure service provision and the related effect of flattening rhythms require temporally structured work. Work for indoor space heating is one of the prime examples of this type of activity. When functioning, the infrastructure for heat provision provides even and flat indoor environments, but this is an achievement that requires work and produces temporal structures of dedicated human action. Such work, we argue, reflects both the rhythmicity of nature and other infrastructures and the ideals of comfort, convenience and detachment from nature.

In the following, we offer a brief account of the organization of wood-based heating work in contemporary Finland. This account is based on a close reading of the diaries collected by the Finnish Literary Society. The diaries are the result of two rounds of diary calls, in 1999 and 2009 , which asked respondents to document a course of a single day - 2 February in both years - as a way to collect and archive contemporary folk culture. These initiatives were highly successful and resulted in more than 30,000 entries documenting the everyday life of different social groups across the country. Of the 1,000 diaries we consulted, ranging between 1-10 pages in length, about 100 deal with wood-based heating (among other things).

While they represent a very traditional way of staying warm, wood-based heating systems remain popular. Wood is the main source of heat in 25 per cent of Finnish detached houses and is also popular in other Nordic countries (Petersen 2008; Nyrud et al. 2008). Even if houses in these regions typically also depend on and make use of other energy sources such as electricity and heating oil, the use of wood structures normal winter days (Jalas \& Rinkinen 2013) and is an important precautionary measure against infrastructure failures.

The practice of heating with wood is, nevertheless, far from simple. It requires the management of two-year cycles of obtaining, drying and using firewood and the operation and maintenance of machinery therein. The management of the flow of the heavy material from various stocks to the final point of use and the lighting of fires and control of registers in the course of the day constitute a weekly and daily rhythm in the analyzed diaries. We also find examples in which longer periods of absence from home require anticipatory activities and the buffering of heat.

To make the practice of using wood as a source of domestic heat viable, the diarists coordinate heating with other activities such as paid work, social activities and health concerns, as well as with weather events. This implies multitasking on various scales: firewood is fetched when collecting the newspaper, fires are lit while coffee gets brewed, neighbourly help in firewood management is given and received, and wood sourcing mingles with outdoor leisure activities and visiting relatives. It also implies routines of sharing work within the household. Wood-based heating is viable, we claim, only as a result of routinized patterns of work in which work is organized into particular sequences of activities that are synchronized with other demands of everyday life (Jalas \& Rinkinen 2013).

In order to achieve an impression of smooth, regular availability and a flattening of rhythms, technological systems and infrastructures need buffers of a different kind. This holds true also for wood-based heating. In the diaries, firewood reserves are stocked to cover the yearly demand and enough wood is stored indoors to provide heat for at least one day. Wood is burned in central boilers and baking ovens, which can store heat for a few days demand. All these buffers imply that the efforts of labour have a frequency, while comfort services can be enjoyed more or less constantly. Some of this buffering is visible in Fig. 6, which depicts the average time spent on heating work according to Finnish time use survey data. In this figure, there is a two-day cycle and, strikingly, a weekly society-wide collective cycle that at least matches - or is driven by - the Sunday sabbatical.

The continued success and wide application of these laboursome and inconvenient systems suggests that the use of wood bears cultural meanings that legitimate the practice. Wood is both a taken-for-granted way of heating a house in Nordic countries and an aesthetically valued practice that carries inherent meanings and provides pleasure for the engaged practitioners (Petersen 2008; Jalas \& Rinkinen 2013). While previous research has highlighted the aesthetics of wood heating, the diary data we analyzed suggests that rhythmicity also plays a pivotal role.

With this short introduction to wood-based heating, we are in a position to make more general claims. The impression of the duration of sameness, the flatness of everyday life, is partly an achievement of properly functioning infrastructures. It is by the nature of infrastructures and the political emphasis on reliability that infrastructures manage to achieve the standardization of everyday life. Yet constant service and the suppression of rhythmicity is a mixed blessing. Jalas and Rinkinen (2013) suggest that the temporal structuring around wood-based heating actually helps to organize the day, create routines and even develop identities. It is against such a notion that we also understand failures in infrastructure services - such electricity blackouts - as rhythm events. That is, failures reveal a rhythmicity that in normal states is hidden by a properly functioning infrastructure. This partly explains the positive accounts of blackouts as moments of doing something different or differently (Trentmann 2009; Silvast 2013).

These two ways of encountering rhythms are of course quite different. Wood-based heating systems require infrastructure work that aims to provide continuity in terms of indoor comfort. It is repetitious and rhythmic work in which gradual coordination with other activities makes the practice viable. It is work that renders the convenience and sustained delivery of heat services as an accomplishment that people take pride in. By 
contrast, infrastructure failures and the subsequent sudden exposure to rhythms are the opposite of everydayness. They are short-lived experiences of the rhythms - adventurous events in which nature gains access to indoor environments.

\section{Conclusion}

In this article we have suggested an approach to energy infrastructures and large-scale technical systems from the perspective of social rhythms. Quite obviously, the availability of critical infrastructure services such as power, water or transportation services affects many other human activities. By the same token, it is obvious that the reliability of infrastructure services are a key political concern.

Infrastructures are ideally opaque and serve us unnoticed. Accordingly, the availability of services neither hinders nor promotes a particular patterning of activities. Rather, ideal infrastructures flatten rhythms that exist in, for example, nature and isolate human activities into a technically mediated sphere.

Infrastructures, however, seldom function ideally. Even when reliable and functioning properly, the possibility that service production may not match peak demands is a significant risk that operators, such as high-voltage electricity companies, anticipate and manage on a routine basis. Congestion and overload are not merely abnormal events, but also rhythms of infrastructure services. Moreover, not all infrastructures are designed for constant operation. Public and private office hours regulate the availability of services according to recurring patterns. We have pushed this notion to claim that recurring weather events such as frost, flooding and rainfall can be understood as rhythmic infrastructures. We also suggested that infrastructures are layered and that a failure in one thus exposes service users to a new, either technical or natural, rhythm.

Reliable infrastructure services are a mixed blessing. While they are obviously critical for the functioning of society and the maintenance of routinized patterns of activity, they also contribute to a sense of monotony. Against this background, we have suggested, blackouts are curiously reported as pleasurable, and as 'making a holiday'. At the same time, our example of wood-based heating suggested another, more regular way of experiencing and constructing rhythmicity in everyday life. Householders who engage in this activity perform heating work to bring about comfortable and stable indoor temperatures. However, the work itself is patterned yearly, weekly and daily according to the needs of the operation and maintenance of these technical systems. Rhythmicity, we proposed, is also one way to understand how such an inconvenient, laboursome and physically demanding system like wood-based heating could be so positively appraised.

We wish to thank the anonymous reviewer and the editors of this special issue for their valuable comments. The work has been financed by the Academy of Finland project 'Smart Energy Transition'.

Adam, B. 1995. Timewatch: The social analysis of time. Cambridge: Polity Press.

Darby, S. 2012. Metering: EU policy and implications for fuel poor households. Energy Policy 49: 98-106.

Edwards, P. 2003. Infrastructure and modernity: Force, time and social organization in the history of sociotechnical systems. In T.J. Misa et al. (eds) Modernity and technology, 185-225. Cambridge, MA: MIT Press.

- et al. 2009. Introduction: An agenda for infrastructure studies. Journal of the Association for Information Systems 10(5): 364-74.

Graham, S. (ed.) 2012. Disrupted cities: When infrastructure fails. London: Routledge.

Hitchings, R., \& S.J. Lee 2008. Air conditioning and the material culture of routine human encasement: The case of young people in contemporary Singapore. Journal of Material Culture 13(3): 251-265.

Hughes, T. 1983. Networks of power: Electrification in Western society, 1880-1930. Baltimore: Johns Hopkins University Press.

- 1989. The evolution of large scale technological systems. In W. Bijker et al. (eds) The social construction of technological systems: New directions in the sociology and history of technology, 51-82. Cambridge, MA: MIT Press.

Jalas, M. \& J. Rinkinen 2013. Stacking wood and staying warm: Time, temporality and housework around domestic heating systems. Journal of Consumer Culture, 11 November.

Lefebvre, H. (1959) 2002. Critique of everyday life, 2: Foundations for a sociology of the everyday. New York: Verso.

- (1974) 1991. The production of space. Oxford: Blackwell Publishing.

Nyrud, A. et al. 2008. Residential bioenergy heating: A study of consumer perceptions of improved woodstoves. Energy Policy 36(8): 3169-3176.

Petersen, L. 2008. Autonomy and proximity in household heating practices: The case of wood-burning stoves. Journal of Environmental Policy \& Planning 10(4): 423-438.

Rinkinen, J. 2013. Electricity blackouts and hybrid systems of provision: Users and the 'reflective practice'. Energy, Sustainability and Society 3(25): 1-10. 
-2015. Demanding energy in everyday life: Insights from wood-heating into theories of social practice. PhD Thesis, Aalto University, Helsinki.

Schwartz Cowan, R. 1983. More work for mother: The ironies of household technology from the open hearth to the microwave. New York: Basic Books.

Shove, E. et al. 2012. The dynamics of social practice: Everyday life and how it changes. London: Sage.

- et al. 2015. Conceptualizing connections: Energy demand, infrastructures and social practices. European Journal of Social Theory 18(3): 274-287.

Silvast, A. 2013. Anticipating interruptions: Security and risk in a liberalised electricity infrastructure. PhD thesis, University of Helsinki.

- et al 2013. Energy in society: Energy systems and infrastructures in society. Science and Technology Studies 26(3): 3-13.

Southerton, D. et al. (eds) 2004. Sustainable consumption: The implications of changing infrastructures of provision. Massachusetts: Edward Elgar Publishing.

Trentmann, F. 2009. Disruption is normal: Blackouts, breakdowns and the elasticity of everyday life. In E. Shove et al. (eds) Time, consumption and everyday life: Practice, materiality and culture, 67-84. Oxford: Berg.

van der Vleuten, E. 2004. Infrastructures and societal change: A view from the large technical systems field.

Technology Analysis \& Strategic Management 16(3): 395-414.

van Vliet, B. et al. 2005. Infrastructures of consumption: Environmental innovation in the utility industries. London: Earthscan.

Fig. 1. An oil burner in the boiler room of a detached house.

Fig. 2. A typical fireplace in the Nordic countries where wood is a popular fuel.

Fig. 3. Firewood stored with protection from the weather.

Fig. 4. Energy consumption puts stress on the grid during cold weather.

Fig. 5. Heating oil storage as part of large-scale energy infrastructure.

Fig. 6. The average time [min/day] spent daily on heating and water provisioning in the period from October-March by Finns living in detached houses (Source: Time use survey data 1998-1999). 